\title{
Serum iron levels increased by cancer chemotherapy correlate the chemotherapy-induced nausea and vomiting
}

\author{
Toshimichi Miya $^{1,2} \cdot$ Haruhiko Kondo ${ }^{1} \cdot$ Akihiko Gemma $^{2}$
}

Received: 26 March 2018 / Accepted: 18 July 2018 / Published online: 24 July 2018

(c) The Author(s) 2018

\begin{abstract}
Background The pathogenesis of chemotherapy-induced nausea and vomiting (CINV) is not fully elucidated. We hypothesized that serum iron levels may be associated with CINV because symptoms of iron poisoning resemble the adverse effects of chemotherapy.

Methods Patients with lung cancer undergoing chemotherapy were included in this retrospective study where serum iron level, unsaturated iron-binding capacity (UIBC), total iron-binding capacity, and ferritin were available prior to and on days 2 and 8 of chemotherapy.

Results Fifty-two patients were analyzed. Iron levels on day 2 were markedly increased in patients receiving highly emetogenic chemotherapy (HEC, $231.0 \pm 45.0 \mu \mathrm{g} / \mathrm{dl}$ ) and moderately emetogenic chemotherapy (MEC, $226.6 \pm 44.2 \mu \mathrm{g} / \mathrm{dl}$ ), and mildly increased in patients receiving low emetogenic chemotherapy (LEC, $104 \pm 51.4 \mu \mathrm{g} / \mathrm{dl}$ ). Significant differences in iron levels on day 2 were observed between the HEC and LEC $(P=0.002)$ and MEC and LEC $(P=0.0007)$ groups. UIBC levels decreased on day $2(18.0 \pm 17.5 \mu \mathrm{g} / \mathrm{dl}$ in HEC, $20.4 \pm 46.8 \mu \mathrm{g} / \mathrm{dl}$ in MEC, and $123.9 \pm 65.9 \mu \mathrm{g} / \mathrm{dl}$ in LEC). There were significant differences in UIBC on days 2 between the HEC and LEC $(P=0.0005)$ and MEC and LEC $(P=0.0015)$ groups. No significant changes in these parameters were observed in a minimal risk group.

Conclusions Iron levels increased according to the emetogenic risk. Accompanied by a markedly increased iron level, nontransferrin bound iron, a highly cytotoxic form of iron, may be present in the serum. Iron removal with an iron-chelating agent may represent a novel antiemetic therapy in patients undergoing chemotherapy.
\end{abstract}

Keywords Chemotherapy-induced nausea and vomiting $\cdot$ Non-transferrin bound iron · Iron overload $\cdot$ Iron poisoning $\cdot$ Iron toxicity

\section{Introduction}

Despite the development of antiemetic treatments including corticosteroids, 5-hydroxytryptamine (5-HT3) receptor antagonists, and neurokinin-1(NK-1) receptor inhibitors, chemotherapy-induced nausea and vomiting (CINV) remains a major adverse toxicity of cancer chemotherapy that reduces the quality of life (QOL) of cancer patients. It is recommended that the antiemetic treatments should be

Toshimichi Miya

tmiya@ks.kyorin-u.ac.jp

1 Department of Thoracic Surgery, Kyorin University Hospital, 6-20-2 Shinkawa, Mitaka, Tokyo 181-8611, Japan

2 Department of Pulmonary Medicine/Medical Oncology, Nippon Medical School, 1-1-5 Sendagi, Bunkyo-ku, Tokyo 113-8603, Japan administered according to the emetogenic risk classification of clinical practice guidelines $[1,2]$; however, the treatments are often suboptimal and CINV remains a persistent problem [3]. The occurrence of CINV is biphasic, comprising acute-phase CINV that occurs within $24 \mathrm{~h}$ and delayed phase CINV that occurs $24 \mathrm{~h}$ or later from the initiation of chemotherapy [1-5]. The two phases of CINV are thought to be mediated by different mechanisms. 5-HT3 receptor antagonists can effectively prevent and control CINV during the acute phase but are generally less effective in the delayed phase [5]. NK-1 inhibitors alleviate the emetic effects of substance $\mathrm{P}$ on the central chemotrigger zone and are effective against CINV in both the acute and delayed phases, suggesting a role for substance $\mathrm{P}$ in the development of CINV in the delayed phase $[6,7]$. However, the pathophysiology of acute and delayed CINV has not yet been fully elucidated, and 
improvements in antiemetic therapy are required to eliminate CINV in all cancer patients undergoing chemotherapy [4].

Acute iron poisoning is a serious toxicity caused by a large excess of iron intake, such as during accidental ingestion by children or individuals attempting suicide, and is the leading cause of death by poisoning in children [8]. Excess iron has a cytotoxic effect in cells and tissues through its ability to catalyze the generation of reactive radicals, producing various symptoms such as nausea, vomiting, diarrhea, liver injury, cardiac disorder, gastroenteritis, and metabolic acidosis $[8,9]$. Serum iron levels are therefore strictly regulated in normal physiological conditions. In the case of iron poisoning, serum iron levels of $1000 \mu \mathrm{g} / \mathrm{dl}$ are typically lethal but symptoms may appear in patients with serum iron levels of approximately $200-250 \mu \mathrm{g} / \mathrm{dl}$ [10]. Serum iron levels have been shown to increase following the administration of various chemotherapeutic agents. Ochiai et al. reported that serum iron levels were increased after chemotherapy comprising leucovorin and fluorouracil plus oxaliplatin (FOLFOX) or leucovorin and fluorouracil plus irinotecan (FOLFIRI) [11], and suggested that increased iron level may be a predictable marker of patients with advanced colorectal cancer. A relationship between serum iron levels and the adverse effects of chemotherapy has not previously been reported. We hypothesized that the symptoms of iron poisoning resemble the adverse effects of chemotherapy, and that increased iron levels could be associated with these adverse effects. Therefore, we investigated the relationship between iron level and CINV in patients receiving cancer chemotherapy.

\section{Patients and methods}

\section{Patient selection}

Retrospective patient data from January 2000 to August 2017 were collected from clinical records at our hospitals. All chemotherapeutic regimens and biochemical tests were performed as standard clinical practice covered by the national health insurance of Japan. Patients with lung cancer receiving cancer chemotherapy were included in this study if serum iron level, unsaturated iron-binding capacity (UIBC), and total iron-binding capacity (TIBC) prior to chemotherapy and on days 2 and 8 were available. All patients had an Eastern Cooperative Oncology Group performance status of 0 to 1 and no severe comorbidities affecting the indication of cytotoxic chemotherapy. Serum iron level, UIBC, and TIBC were measured as part of routine blood analysis where practically necessary. The measurement of iron was not scheduled for the majority of patients, resulting in a prolonged data collection period and limited sample size. Treatment regimens were classified as highly emetogenic chemotherapy
(HEC), moderately emetogenic chemotherapy (MEC), low emetogenic chemotherapy (LEC), and minimum risk chemotherapy (MIC) according to clinical practice guidelines of the Multinational Association of Supportive Care in Cancer (MASCC) and the European Society of Medical Oncology (ESMO) [1]. For MIC patients, data from day 2 were not obtainable because blood laboratory testing is typically not performed in patients treated with MIC. Furthermore, biochemical data from day 8 or later are designated only as day 8 because there is thought to be little difference in the levels of iron, UIBC, and TIBC between day 8 and later days in patients daily taking tyrosine kinase inhibitors. Changes in serum iron, UIBC, and TIBC during treatment were investigated to determine whether they correlated with the emetic risk of chemotherapy.

This study has been approved by the Institutional Review Board of Kyorin University.

\section{Statistical analysis}

Statistical analyses were performed using the Mann-Whitney $U$ test for unequal variances. Values of $P<0.05$ were considered statistically significant.

\section{Results}

A total of 52 patients (male 34/female 18) were included in the analysis (Table 1). All patients had an Eastern Cooperative Oncology Group performance status of 0 to 1 . The number of patients in each group was 20 in HEC, 14 in MEC, 7 in LEC, and 11 in MIC. The regimens administered in each group contained cisplatin in HEC; carboplatin, irinotecan, and amrubicin in MEC; pemetrexed, docetaxel, and gemcitabine in LEC; and gefitinib and osimertinib in MIC group.

Baseline levels of serum iron and UIBC prior to chemotherapy did not differ between the groups (Table 2; Fig. 1). However, iron levels were markedly increased on day 2 in HEC $(231.0 \pm 45.0 \mu \mathrm{g} / \mathrm{dl})$ and MEC $(226.6 \pm 44.2 \mu \mathrm{g} / \mathrm{dl})$ and mildly increased in LEC $(104 \pm 52.4 \mu \mathrm{g} / \mathrm{dl})$ groups. There was a significant difference in iron levels on day 2 between the HEC and LEC $(P=0.002)$ and the MEC and LEC $(P=0.0007)$ groups. On day 8 , iron levels remained high in the HEC group and had a tendency to decrease in the MEC group. In contrast, iron levels had returned to normal in the LEC group by day 8. A significant difference in iron levels was observed on day 8 between the HEC and LEC $(P=0.0002)$, HEC and MIC $(P=0.0001)$, MEC and LEC $(P=0.0009)$, and MEC and MIC $(P=0.032)$ groups.

With increased iron, UIBC levels decreased accordingly on day $2(18.0 \pm 17.5 \mu \mathrm{g} / \mathrm{dl}$ in HEC, $20.4 \pm 46.8 \mu \mathrm{g} /$ $\mathrm{dl}$ in MEC, and $123.9 \pm 65.9 \mu \mathrm{g} / \mathrm{dl}$ in LEC) and day 8 $(30.2 \pm 54.7 \mu \mathrm{g} / \mathrm{dl}$ in HEC, $75.7 \pm 76.0 \mu \mathrm{g} / \mathrm{dl}$ in MEC, and 
Table 1 Patient characteristics and chemotherapy regimens

\begin{tabular}{ll}
\hline & No. of patients \\
\hline All patients & \\
Total/male/female & $52 / 34 / 18$ \\
Median age, years (range) & 65 (41-87) \\
Lung cancer & 52 \\
Tumor type & \\
HEC & \\
Total/male/female & $20 / 15 / 5$ \\
CDDP/etoposide & 14 \\
CDDP/vinorelbine & 6 \\
MEC & \\
Total/male/female & $14 / 9 / 5$ \\
CBDCA/gemcitabine & 5 \\
CBDCA/paclitaxel & 2 \\
CBDCA/etoposide & 2 \\
CBDCA/pemetrexed & 2 \\
Irinotecan & 2 \\
Amrubicin & 1 \\
LEC & \\
Total/male/female & $7 / 6 / 1$ \\
Pemetrexed & 5 \\
Docetaxel & 1 \\
Gemcitabine & 1 \\
MIC & \\
Total/male/female & \\
Gefitinib & \\
Osimertinib & \\
\hline & \\
\hline &
\end{tabular}

$H E C$ highly emetogenic chemotherapy, $M E C$ moderately emetogenic chemotherapy, LEC low emetogenic chemotherapy, MIC minimum risk chemotherapy, $C D D P$ cisplatin, $C B D C A$ carboplatin

$123.9 \pm 65.9 \mu \mathrm{g} / \mathrm{dl}$ in LEC). There were also significant differences in the levels of UIBC between the HEC and LEC $(P=0.0005)$ and MEC and LEC $(P=0.0015)$ groups on day
2, and between the HEC and LEC $(P=0.0009)$, HEC and MIC $(P=0.0001)$, MEC and LEC $(P=0.0017)$, and MEC and MIC $(P=0.0013)$ groups on day 8 (Table 2; Fig. 1). There was no apparent change in iron level, UIBC, or TIBC in the MIC group from baseline to day 8 .

The saturation ratio of TIBC [calculated as saturation ratio $(\%)=$ serum iron/TIBC $\times 100]$ increased to 92.1 and $96.5 \%$ on day 2 and 95.1 and $72.3 \%$ on day 8 in the HEC and MEC groups, respectively.

\section{Discussion}

The emergence of new therapeutic options including 5-HT3 antagonist and an NK-1 receptor inhibitor have revolutionized the prevention of CINV [12]. However, even with modern antiemetic strategies, more than half of patients receiving HEC or MEC are affected by CINV $[3,7,13]$. Since the aim of antiemetic therapy is to minimize or eliminate CINV in all patients, current clinical options are insufficient and novel approaches to reduce CINV should be investigated.

In a healthy individual, most serum iron is bound to transferrin and the remaining non-transferrin bound iron (NTBI) fraction is extremely small $[14,15]$. NTBI is potentially a toxic chemical, given its high propensity to induce reactive oxygen species, and is responsible for cellular damage producing gastroenteritis, cardiac disease, and liver injury [14-19]. Gastrointestinal toxicity is a well-known adverse effect of oral iron therapy [19]. Patients administered iron tablets for iron deficiency anemia frequently suffer from nausea or diarrhea [18], with even a small quantity of NTBI released from iron tablets likely to cause adverse effects. In the case of iron poisoning resulting from an overdose of iron tablets, gastrointestinal toxicities such as nausea and vomiting appear in patients with maximum serum iron levels of $350-500 \mu \mathrm{g} / \mathrm{dl}$, and metabolic acidosis subsequently occurs as a result of free radical ions of iron [17]. Systemic toxicity
Table 2 Changes in serum levels of iron and UIBC according to emetogenic classification

\begin{tabular}{|c|c|c|c|c|c|c|c|}
\hline & \multirow[t]{2}{*}{$n$} & \multicolumn{3}{|c|}{ Serum iron $(\mu \mathrm{g} / \mathrm{dl})$} & \multicolumn{3}{|l|}{ UIBC $(\mu \mathrm{g} / \mathrm{dl})$} \\
\hline & & Baseline & Day 2 & Day 8 & Baseline & Day 2 & Day 8 \\
\hline HEC & 20 & $61.7 \pm 10.8$ & $231.0 \pm 45.0$ & $217.3 \pm 66.5$ & $193.5 \pm 44.0$ & $18.0 \pm 17.5$ & $30.2 \pm 54.7$ \\
\hline MEC & 14 & $68.6 \pm 5.6$ & $226.6 \pm 44.2$ & $168.6 \pm 88.1$ & $206.1 \pm 42.5$ & $20.4 \pm 46.8$ & $75.7 \pm 76.0$ \\
\hline LEC & 7 & $51.9 \pm 16.0$ & $104.4 \pm 51.4$ & $36.5 \pm 21.0$ & $191.1 \pm 53.1$ & $123.9 \pm 65.9$ & $170.3 \pm 24.9$ \\
\hline $\mathrm{MIC}$ & 11 & $61.0 \pm 43.3$ & NA & $73.4 \pm 25.2$ & $263.8 \pm 72.1$ & NA & $255.3 \pm 75.4$ \\
\hline
\end{tabular}

Baseline prior to treatment, $H E C$ highly emetogenic chemotherapy, $M E C$ moderately emetogenic chemotherapy, $L E C$ low emetogenic chemotherapy, $M I C$ minimum risk chemotherapy, NA not applicable

Statistically significant differences in iron levels were observed between HEC and LEC $(P=0.002)$ and MEC and LEC $(P=0.0007)$ on day 2 , and between HEC and LEC $(P=0.0002)$, HEC and MIC $(P=0.0001)$, MEC and LEC $(P=0.0009)$, and MEC and MIC $(P=0.031)$ on day 8

Statistically significant differences in UIBC levels were observed between HEC and LEC $(P=0.0005)$, and MEC and LEC $(P=0.0015)$ on day 2 , and between HEC and LEC $(P=0.0009)$, HEC and MIC $(P=0.0001)$, MEC and LEC $(P=0.017)$, and MEC and MIC $(P=0.013)$ on day 8 

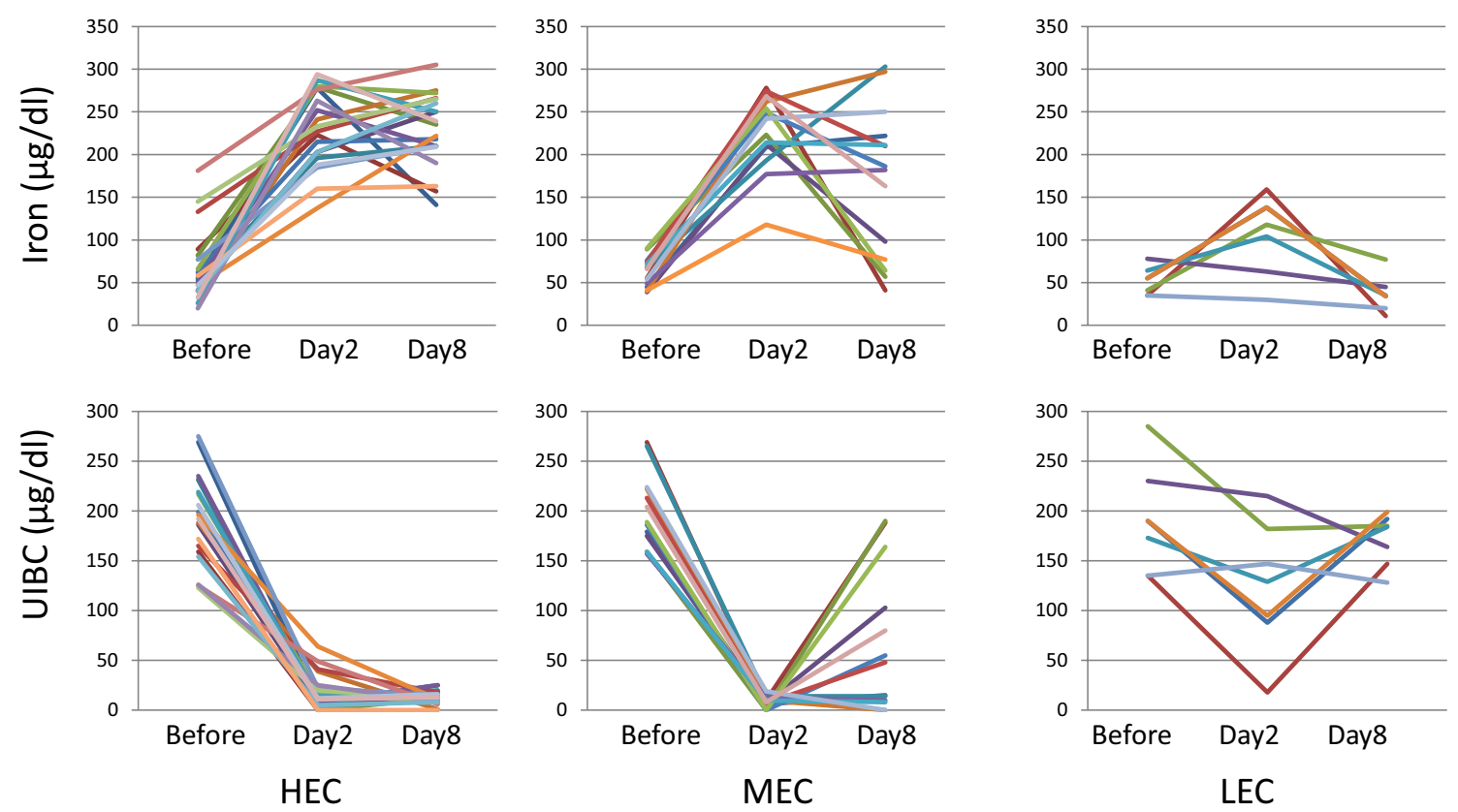

Fig. 1 Changes in serum levels of iron and UIBC according to emetogenic classification. Iron levels were markedly increased on day 2 in the HEC and MEC groups, and mildly increased in the LEC group. Iron levels remained high in HEC, tended to decrease in MEC, and returned to normal levels in the LEC group on day 8. The UIBC lev- els changed accordingly in the HEC, MEC, and LEC groups. Baseline prior to treatment, $H E C$ highly emetogenic chemotherapy, $M E C$ moderately emetogenic chemotherapy, $L E C$ low emetogenic chemotherapy, UIBC unsaturated iron-binding capacity

delayed phases. In contrast, iron levels were less elevated in patients treated with LEC and MIC compared with HEC and MEC. The elevation of iron observed in LEC patients on day 2 and was mild, and no significant elevation on day 8 or later was observed in patients treated with MIC consisting of tyrosine kinase inhibitors. These findings suggest that the degree of elevation of iron level seemed to be related to emetogenic risk classification.

There were several limitations to this study. First, this was a retrospective analysis and the measurement of iron was not routinely scheduled, but was instead performed in a small fraction of patients where practically necessary. Therefore, a long data collection period of over 17 years was required, and the sample size was limited. Despite these limitations, the tendency for increased iron levels to correlate with emetogenicity was evident. It should be emphasized serum iron levels were markedly increased in all patients who received HEC or MEC with no exception whether patients suffering anemia or not. It is definitely evident that iron levels were always increased by cytotoxic chemotherapy and the increased levels reached toxic range in some patients.

The mechanism by which chemotherapy is associated with elevated iron level remains unclear. One-third of iron in the human body is pooled as ferritin or hemosiderin in the liver, the spleen, and the kidneys [20]. Cytotoxic chemotherapy damages normal organs and iron may be released from pooled iron, although serum ferritin levels do not change ous toxicities including nausea and vomiting in the acute and 
significantly with various chemotherapeutic regimens $[10$, 21]. Ochiai et al. suggested that the elevation of serum iron during chemotherapy may be secondary to reduced iron consumption by erythropoiesis, leading to increased expression of hepcidin-25 and suppression of IL-6 via negative feedback [21].

In summary, we found that iron levels increased with chemotherapy according to the grade of emetogenicity. No studies to date have reported a relationship between increased iron levels and adverse effects during chemotherapy. The influence of serum iron on the adverse effects of cancer chemotherapy, in particular, when conducting clinical trials of new anticancer drugs, should therefore be investigated. This phenomenon may represent a novel approach to antiemetic treatment, as iron removal therapy using, for example, an iron-chelating drug may potentially alleviate CINV. We plan to conduct a prospective study to assess the influence of NTBI and the effect of deferoxamine as an iron-chelating drug on CINV and QOL of patients receiving cancer chemotherapy.

\section{Compliance with ethical standards}

Conflict of interest The authors have declared no conflicts of interest.

Open Access This article is distributed under the terms of the Creative Commons Attribution 4.0 International License (http://creativeco mmons.org/licenses/by/4.0/), which permits unrestricted use, distribution, and reproduction in any medium, provided you give appropriate credit to the original author(s) and the source, provide a link to the Creative Commons license, and indicate if changes were made.

\section{References}

1. Roila F, Molassiotis A, Herrstedt J et al (2016) 2016 MASCC and ESMO guideline update for the prevention of chemotherapyinduced and radiotherapy-induced nausea and vomiting and of nausea and vomiting in advanced cancer patients. Ann Oncol 27(suppl5):v119-v133

2. Basch E, Prestrud AA, Hesketh PJ et al (2011) Antiemetics: American Society of Clinical Oncology Clinical Practice guideline update. J Clin Oncol 29:4189-4198

3. Di Maio M, Gallo C, Leighl NB et al (2015) Symptomatic toxicities experienced during anticancer treatment: agreement between patient and physician reporting in three randomized trials. J Clin Oncol 33:910-915

4. Navari RM, Aapro M (2016) Antiemetic prophylaxis for chemotherapy-induced nausea and vomiting. N Engl J Med 374:1356-1367
5. Saito M, Aogi K, Sekine I et al (2009) Palonosetron plus dexamethasone versus granisetron plus dexamethasone for prevention of nausea and vomiting during chemotherapy: a double-blind, double-dummy, randomized, comparative phase III trial. Lancet Oncol 10:115-124

6. Tanioka M, Kitano A, Matsumot K et al (2013) A randomized, placebo-controlled, double-blind study of aprepitant in nondrinking women younger than 70 years receiving moderately emetogenic chemotherapy. Br J Cancer 109:859-865

7. Rojas C, Slusher BS (2012) Pharmacological mechanism of 5-HT3 and tachykinin NK1 receptor antagonist to prevent chemotherapy-induced nausea and vomiting. Eur J Pharmacol 686:1-7

8. Chang TP, Rangan C (2011) Iron poisoning. A literature-based review of epidemiology, diagnosis, and management. Pediatr Emerg Care 27:978-985

9. Papanikalaou G, Papanikolaou K (2005) Iron metabolism and toxicity. Toxicol Appl Pharmacol 202:199-211

10. Anderson GJ (1999) Non-transferrin-bound iron and cellular toxicity. J Gastroenterol Hepatol 14:105-108

11. Ochiai T, Nishimura K, Watanabe T et al (2013) Serum iron levels as a new biomarker in chemotherapy with leucovorin and fluorouracil plus oxaliplatin or leucovorin and fluorouracil plus irinotecan, with or without molecularly-targeted drugs. Mol Clin Oncol $1: 805-810$

12. Aapro M (2018) CINV: still troubling patient after all these years. Support Care Cancer 26(Suppl 1):5-9

13. Ihbe-Heffinger A, Ehlken B, Bernard R et al (2004) The impact of delayed chemotherapy-induced nausea and vomiting on patients, health resource utilization and costs in German Cancer Centers. Ann Oncol 15:526-536

14. Stiff JE, Meldon SW, Tomassoni AJ (1999) Usefulness of the total iron binding capacity in the evaluation and treatment of acute iron overdose. Ann Emerg Med 33:73-76

15. Anderson GJ (1999) Non-transferrin-bound iron and cellular toxicity. J Gastroenterol Hepatol 14:105-108

16. Chattopadhyaya R, Goswami B (2012) Oxidative damage to DNA constituents by iron-mediated Fenton reactions: the deoxyadenosine family. J Biomol Struct Dyn 30:394-406

17. Brissot P, Ropert M, Le Lan C et at (2012) Non-transferrin bound iron: a key role in overload and iron toxicity. Biochim Biophys Acta G Gen Subj 1820:403-410

18. Patel M, Ramavataram DVSS (2012) Non transferrin bound iron: nature, manifestations and analytical approaches for estimation. Ind J Clin Biochem 27:322-333

19. Klein-Schwartz W, Oderda GM, Gorman RL et al (1990) Assessment of management guidelines. Acute iron ingestion. Clin Pediatr (Phila) 29:316-321

20. Siah CM, Ombiga J, Adams LA et al (2006) Normal iron metabolism and the pathophysiology or iron overload disorders. Clin Biochem Rev 27:5-16

21. Ochiai T, Nishimura K, Watanabe T et al (2014) Mechanism underlying the transient increase of serum iron during FOLFOX/ FOLFIRI therapy. Mol Clin Oncol 2:968-972 